JOURNAL

of Health Inequalities

\title{
Alcohol consumption and burden of alcohol- related diseases in Lithuania
}

\author{
Mindaugas Stelemekas \\ Health Research Institute, Faculty of Public Health, Lithuanian University of Health Sciences, Kaunas, Lithuania
}

ADRRESS FOR CORRESPONDENCE: Mindaugas Štelemékas, Health Research Institute, Faculty of Public Health, Lithuanian University of Health Sciences, Kaunas, Lithuania; e-mail: mindaugas.stelemekas@lsmuni.It

Supplementary materials (slides from the conference) are available in Webbappendix at the Journal's website: https://www.termedia.pl/ Journal/Journal_of_Health_Inequalities-100

For many years alcohol consumption in Lithuania has been widely identified as a major challenge for health in the society, and major changes in alcohol control legislation has started in 2008-2009 when excise taxation has been increased, alcohol advertising has been limited during daytime, night off-premise sales of alcohol has been banned, and drink-driving legislation significantly toughened. The next period of new alcohol control measures took place in 2014-2018 when excise taxation has been increased multiple times (including doubling of excise for beer and wine in 2017), alcohol sales in petrol stations has been banned since 2016. Since 2018 a legal alcohol purchase age has been increased from 18 to 20 years of age, and a near full alcohol advertising ban introduced in all social and media outlets.

The policies implemented during those two periods may be associated with a significant impact to health indicators of Lithuanian population. Life expectancy at birth from 2007 to 2019 has increased by 5.7 years, followed by significant declines in many alcohol related indicators. For instance, in 2019 a standardized alcohol attributable mortality indicator has been 3 times lower comparing to a year 2007 (a decline from 57.8 deaths per 100000 in 2007 to 16.8 deaths per 100000 in 2019). The more detailed analysis of the effects of alcohol control policies have identified that the all-cause mortality has declined by about $3 \%$ more during periods when alcohol control policies were introduced comparing with the periods without new policies.

According to WHO estimates in 2016 Lithuania was a country consuming the most alcohol in the European Region. However, the most significant changes per capita alcohol consumption in Lithuania was also observed in recent years when in 2015 a recorded alcohol consumption among 15 years old and older populations was 14 liters and it has declined to 11.1 liters in 2019. It is important to note, that despite the positive declining trends of recorded alcohol consumption and alcohol related mortality indicators, the budgetary income from excise taxation has increased, especially during the last few years (the excise income has increased by $44 \%$ from 2016 to 2019).

In summary, Lithuania sets an example in the field of public health showing how strong alcohol control policies (including WHO "best buys") may significantly contribute in improving multiple public health indicators in a relatively short period of time.

Key words: alcohol control, alcohol consumption, alcohol-related diseases.

\section{DISCLOSURE}

The author reports no conflict of interest. 